\title{
3D Face Recognition: Feature Extraction based on Directional Signatures from Range Data and Disparity Maps
}

\author{
Soodamani Ramalingam \\ School of Engineering and Technology \\ University of Hertfordshire \\ College Lane Campus, Hatfield, Herts AL10 9AB, UK \\ Email: s.ramalingam@ herts.ac.uk
}

\begin{abstract}
In this paper, the author presents a work on i) range data and ii) stereo-vision system based disparity map profiling that are used as signatures for 3D face recognition. The signatures capture the intensity variations along a line at sample points on a face in any particular direction. The directional signatures and some of their combinations are compared to study the variability in recognition performances. Two $3 \mathrm{D}$ face image datasets namely, a local student database captured with a stereo vision system and the FRGC v1 range dataset are used for performance evaluation.
\end{abstract}

Keywords-3D Face Recognition, feature extraction, directional signatures, FRGC, performance evaluation, ROC.

\section{INTRODUCTION}

Research in 3D Face Recognition systems is becoming increasingly popular due to the development of more affordable 3D image acquisition systems and the availability of 3D face databases. Such systems have made quite a progress in solving problems of localisation, pose and illumination variances. However, these problems still continue to exist. With security applications such as Border Crossing, it is difficult to acquire idealistic images without being constrained and intrusive at capture points.

In 3D profile generation techniques, only one angle of planar intersection with the 3D image is typically considered. Such techniques deal with variations in pose by normalising the image to a standard pose as a preprocessing step. Additionally, automatic illumination normalisation techniques do not reach an optimal performance with uniformity across a database. This has been experimented on the FRVT database on 4 different illumination normalisation techniques namely global equalization, parabola equalization, double equalization \& wavelet-based equalization [18] as indicated by legends $g, p, d$ and $w$ respectively in Fig.1. The double equalisation algorithm is the only one that had managed to achieve an SSE of zero at some points, but not across the board. There is a residue despite normalisation. Face recognition technologies have to cope and perform under such noisy environments.

In this paper, the author chooses to determine the $3 \mathrm{D}$ profile called signatures along several possible angles of planar intersections with the $3 \mathrm{D}$ image to accommodate such illumination and small $2 \mathrm{D}$ pose variations. For a chosen angle, a set of 3D signatures are derived along the $\mathrm{Y}$-axis at fixed sampling points. Variations in angles include $0^{\circ}, 45^{\circ}, 90^{\circ}, 135^{\circ}$ and their combinations of $0^{\circ}+45^{\circ}$, $135^{\circ}+45^{\circ}, 135^{\circ}+45^{\circ}+0^{\circ}, 90^{\circ}+45^{\circ}, 90^{\circ}+135^{\circ}, 90^{\circ}+135^{\circ}+0^{\circ}$, $90^{\circ}+135^{\circ}+45^{\circ}, 135^{\circ}+0^{\circ}$. From these signatures, statistical moments are determined as feature sets. Performance evaluations through ROC for the above modalities have been carried out using the following experimental setup:

\section{A. Databases:}

a) Student Database- $D B_{1}$ :

A student database captured from a Stereo Vision Systems [1] consisting of the 100 students as subjects with 10 canonical views per subject (fixed sample sizes) under a controlled illumination environment. Small variations in pose were allowed. The canonical views span $180^{\circ}$ and therefore an approximate $18^{\circ}$ separation between two consecutive samples.

b) FRVT Database- $\mathrm{DB}_{2}$ :

FRVT data consisting of 275 subjects with varying sample sizes leading to a total of 943 images. The Database consists only of frontal images. The images vary in illumination and scaling. 


\section{B. Model Representations:}

a) Average Model $-M_{\text {avg }}$ With the student DB, an average image is generated from the sample sets of fixed sizes (i.e. number of samples/subject $=k$, constant).

b) Individual Model - $M_{\text {ind }}$ : With FRGC dataset, both average and individual face models are derived and tested separately. The average models were built based on varying sample sizes.

Results show that the features extracted provide good discriminating ability between classes.

The rest of the paper is organized as follows: Section II provides a literature review of 3DFR systems from a feature extraction perspective. Section III details the proposed system. Section IV describes the experimental setup and reports on various performance measures and relative performances of the angular features extracted. Section V provides a conclusion and recommendations for further work.

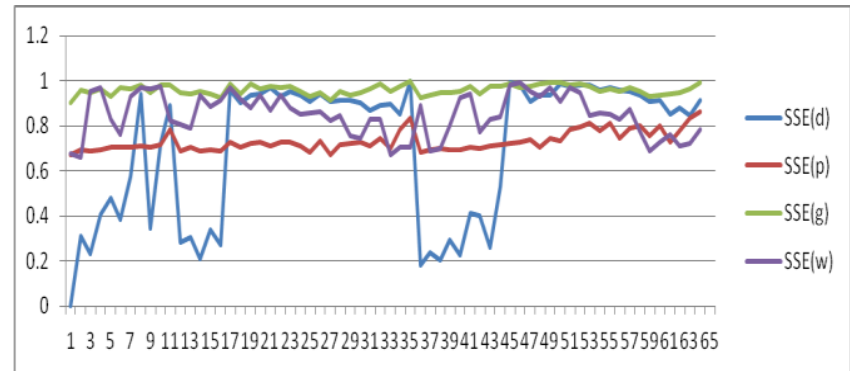

Figure 1: Illumination Normalisation - sum squared error (SSE) plot fo four different techniques for random samples of the FRVT DB

TABLE I. FRVT SAMPLE SIZE FREQUENCIES

\begin{tabular}{|c|l|l|l|l|l|l|l|l|}
\hline Sample size & 1 & 2 & 3 & 4 & 5 & 6 & 7 & 8 \\
\hline Frequency & 77 & 32 & 47 & 33 & 28 & 30 & 15 & 13 \\
\hline
\end{tabular}

\section{Review Of CuRrent Position}

The field of 3D Face Recognition (3DFR) is quite new but advancing quite rapidly. At the algorithmic level, the techniques vary depending on the modes of model representation (or registration), feature extraction and matching. Feature extraction has recently gained a lot of prominence as it dictates the performance of a recognition system. A good set of survey papers [1-2] provide varied systems on generic 3DFR. These cover a range of techniques starting from imaging, representation, matching, both grey scale as well as colour images. In this section, we consider a brief review of current techniques that is related to $3 \mathrm{D}$ facial feature extraction.

The popular idea of using local shape descriptors extend from 2D to 3D [3] making sparse representations of face models feasible [4-5]. Even though research in 3DFR claim having solved problems of pose invariance as compared to $2 \mathrm{D}$, most research work in $3 \mathrm{D}$ continues to focus on pose invariance [6-7]. It is well acknowledged that face recognition systems under perform as a single modality. The success of multi-modal systems and in particular $2 \mathrm{D}+3 \mathrm{D}$ face recognition algorithms are becoming a popular but simpler approach to improving recognition accuracies [7-8]. In [3], Wang et al utilise 3D+2D image features and fuse final recognition using PCA which showed improved performance in comparison to single modal systems. Such systems typically require manual selection of fiducial points for pose normalisation. In addition, matching requires perfect image alignments and filling missing points through interpolation.

The marriage of image processing and computer graphics provides robust performance under noisy conditions by use of morphable models [9]. An emerging area is that of geodesic distance measurement [7], which is the shortest distance between two points, is a good candidate for feature extraction. Geodesic distances provide a basis for mapping 3D space into a 2D image. These approaches assume that human face is isometric, which indicates the preservation of geodesic distance in various expressions. Moments are used as features and treated as a face signature in [10].

The work by Gorden [11] uses disparity maps to model faces is similar to the approach adopted in the proposed work here. The author employs curvature estimations on range data along with depth information for face recognition. The paper reports high accuracy (70-100\%) and viewpoint invariance. Lee and Milos [22] segment range images into convex regions based on the signs of mean and Gaussian curvatures leading to an Extended Guassian Image (EGI).

Beumier and Acheroy[13] derive a 3D facial structure and its information is used for recognition. The process requires pose normalisation and extracting profile curves at the intersections of facial surface with evenly spaced vertical planes. A 3D face database of size 120 with 30 people was tested giving an EER of $9-13 \%$ when automatic normalisation is used and an EER $3.25-6 \%$ when manual orientation is used.

In [14], Razden et al., have a combined feature extraction, facial profile signatures, and partial surface for matching of triangular meshes. Surface classification based on mean and Gaussian curvatures is followed. Their approach was tested on 117 people with 421 scans of varying facial expressions captured at the PRISM lab at Arizona State University. Their reported authentication performance is an EER of $0.065 \%$ for normal faces and $1.13 \%$ for faces with expressions. Verification results of $100 \%$ in normal faces with expressions at $0.1 \%$ FAR. For 
identification, the performance was $100 \%$ in normal faces and $95.6 \%$ with expressions.

The Face Recognition Vendor Technology (FRVT) 2006 includes a sequestered evaluation of 3DFR systems conducted for the first time in 2006 from high resolution still and 3D imagery collected from controlled environment[15] [Fig2]. A key measure of performance is the False Reject Rate (FRR) at a False Accept Rate (FAR) of 0.001. These high performance rates have been contributed to the algorithm design that takes advantage of the image size and quality. Performance in FRVT 2006 shows an order of magnitude increase. Two of Viisage's algorithms ranked the first in FRVT 2006 evaluation followed by Geometrix and University of Houston.

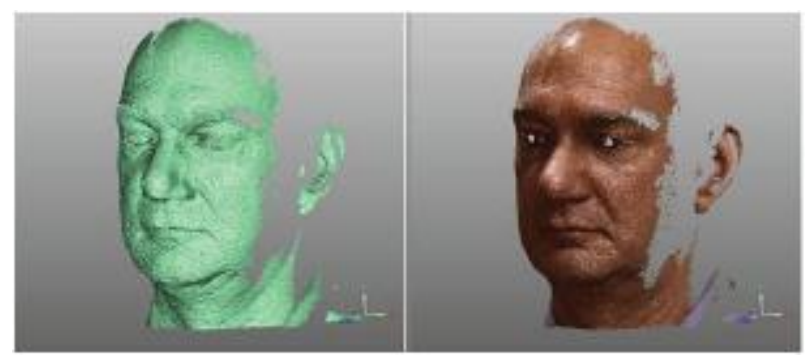

Figure 2. Example of FRVT 2006 3D dataset - shape and texture channles respectively.[15]

In this paper, the author derives 3D profiles called signatures at regular intervals on the face at points of intersection with a plane. This approach is similar to that of Beumier and Acheroy but the key difference is that a set of signatures corresponding to varied angles of intersection of the plane with the facial image. In this sense, multi-modal signatures from various angles of intersection may conveniently be used to improve performance as shown in this paper. In this process, modelling of facial features from various angles allows for variations in pose to be taken into consideration at the feature extraction level.

\section{PROPOSED SYSTEM}

In this Section, a 3DFR system architecture using facial directional signatures is outlined. The generic block diagram for the system is shown in Fig.4.

\section{A. Data Acquisition}

The proposed 3DFR system deals with databases namely the student database $\left(\mathrm{DB}_{1}\right)$ and FRVT v1 database $\left(\mathrm{DB}_{2}\right)$, samples of which are shown in Fig.3. The student $\mathrm{DB}$ is a disparity map derived from a stereo-vision pair of left and right images. The shape channels were used in case of FRVT dataset. Details of acquisition and mapping into corresponding $2.5 \mathrm{D}$ disparity maps and range data are found in $[15,17]$ respectively for the two databases.

\section{B. Image Normalisation}

For this paper, the student database has two datasets based on the camera lens used namely $7.5 \mathrm{~mm}$ and 12.5 mm. Each partition contains 100 subjects with 10 canonical views per subject with a total of 1000 images in each dataset. The FRVT database consists of frontal images of 275 subjects with varying samples/subject as shown by the frequency distribution in Table I. The size of the database is 943. Both the student and FRVT databases were manually cropped and resized to an image size of $128 \times 128$ pixels. The student DB was acquired in an illumination controlled environment; hence did not require further normalization. The FRVT database required illumination normalisation using the standard histogram equalisation technique available in MATLAB. Thus the DBs were normalised with respect to scaling and illumination (Fig.4). The rest of the steps are common for both databases.
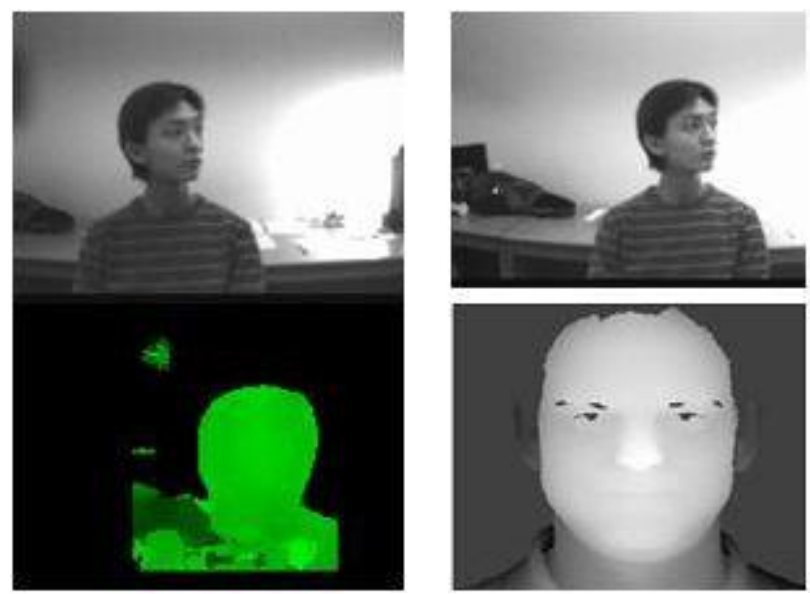

Figure 3: Top Row - Stereo Pair; Bottom Left - Corresponding Disparity Map. Bottom Right - FRVT 2.5 Range Image[15]

\section{3D Profile Signatures}

With the DB images, signatures were derived at the intersections of facial surface with evenly spaced vertical planes. The signatures act as profile curves at sample points along the $\mathrm{Y}$-axis $\left(90^{\circ}\right)$ of the image. For convenience, a fixed set of 40 signatures is derived for each image. Similarly, other directional signatures are also derived, as shown in (Fig.4-5). The 3D signatures appear as a compressed image (Figs.4-5) due the effect of sampling in 3D. Sampling takes place at points of intersection of a stack of planar surfaces oriented in a particular angle with the images.

It's the aim of this paper to evaluate the performance of the system by using these directional signatures as features.

\section{Model Representation}

Models are built to form a feature database suitable for matching. Two approaches are followed: a) an average model constructed by averaging the normalised canonical views as in the student database. b) individual images retained as models of face images. The individual models are useful when there are insufficient samples for the subjects as in the case of the FRVT dataset where the number of samples/subject is one for some part of the 
database (Table I). The within-class distance is larger in the former case compared to the latter as it is a fuzzy representation encompassing the average information from all of the samples of a subject. Therefore, with the average model representation, it is not expected to produce a $100 \%$ match score between the query and the target images even for Validation tests. However, this does not imply that it is a poor representation as it allows an implicit modelling of imprecision within the dataset.

Thus the second aim of this paper is to evaluate the performance of the system based on the above two model representations.

Basic variations in intersecting planar angles with an image include $0^{\circ}, 90^{\circ}, 45^{\circ}$ and $135^{\circ}$ and the corresponding signatures are used as uni-modal features. In addition, concatenated feature sets derived by combining signatures at two or more intersecting angles. The full set comprises of the following combinations:

a) Uni-modal: $\Theta_{1} \in{ }^{1} \mathrm{C}_{4}=\{0,45,90,135\}$

b) Two-angles: $\Theta_{2} \in{ }^{2} \mathrm{C}_{4}=\{0+45,0+90,45+90$, $0+135,45+135,90+135\}$

c) Three-angles: $\Theta_{3} \in{ }^{3} \mathrm{C}_{4}=\{0+45+90,0+45+135$, $0+90+135,45+90+135\}$

d) Four-angles: $\Theta_{4} \in{ }^{4} \mathrm{C}_{4}=\{0+45+90+135\}$

\section{E. Feature extraction}

For the above models, statistical features namely; a set of 7 central moments $[10,16]$ is derived.

$$
\begin{aligned}
\text { Let } x & \rightarrow \text { Number of subjects } \\
y & \rightarrow \text { Samples/subject } \\
\partial & \rightarrow \text { Sampling rate along a chosen axis } \\
\Delta & \rightarrow \text { Length of signature along the other axis } \\
\Theta & \in\left\{{ }^{\mathrm{n}} \mathrm{C}_{\mathrm{r}}\right\} \mid \text { where } n=4 \text { and } r \in\{1,2,3,4\} \\
\text { and } \mu & \rightarrow \text { Central Moments on } \Theta
\end{aligned}
$$

Then, the dimensionality of the feature set is given by

$$
x^{*} y^{*} \partial * \Delta * \mu * \Theta
$$

The dimension of the feature sets for the above model representations for a directional signature along $\mathrm{X}$ or $\mathrm{Y}$ axis is given in Table II. $(\partial, \Delta)$ pair are constants for directional signatures along $\left(0^{\circ}, 90^{\circ}\right)$ respectively. However, in the directions of $45^{\circ}$ and $135^{\circ}$, the value of $\Delta$, i.e. the length of the signature along the diagonals will be larger compared to $\mathrm{X}$ and $\mathrm{Y}$ axes. Further, $\Theta$, the number of directional combinations proportionally increase the feature set dimension.

\section{F. Recognition}

Recognition is carried by Fischer's Linear Discrimant Analysis (FLDA) [1,9]. Given a query image Q, matching requires that $\mathrm{Q}$ undergoes all pre-processing and feature extraction process. For a specific angle of intersection, $Q$ is represented by a feature set defined by $\partial * \Delta * \mu$. That is, by a feature set of dimensionality 40 signatures x 128 (length of each signature) x 7 central moments. Experimental set up and results of recognition are discussed in detail in Section IV.

\begin{tabular}{|c|c|c|c|c|c|c|c|}
\hline $\begin{array}{l}\text { Model } \\
\text { Represen } \\
\text { attion } \downarrow\end{array}$ & $\begin{array}{l}\text { DB } \\
\downarrow\end{array}$ & $x$ & $y$ & $\partial$ & $\Delta$ & $\mu$ & $\Theta$ \\
\hline \multirow{2}{*}{$\begin{array}{c}\text { Average } \\
\text { from } \\
\text { Samples }\end{array}$} & $\begin{array}{c}\text { Student } \\
\text { DB }\end{array}$ & 100 & 1 & 40 & 128 & 7 & \multirow{3}{*}{ 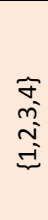 } \\
\hline & $\begin{array}{c}\text { FRVT } \\
\text { DB }\end{array}$ & 275 & 1 & 40 & 128 & 7 & \\
\hline $\begin{array}{l}\text { Individual } \\
\text { Samples }\end{array}$ & $\begin{array}{l}\text { FRVT } \\
\text { DB }\end{array}$ & 275 & {$[1,8]$} & 40 & 128 & 7 & \\
\hline
\end{tabular}

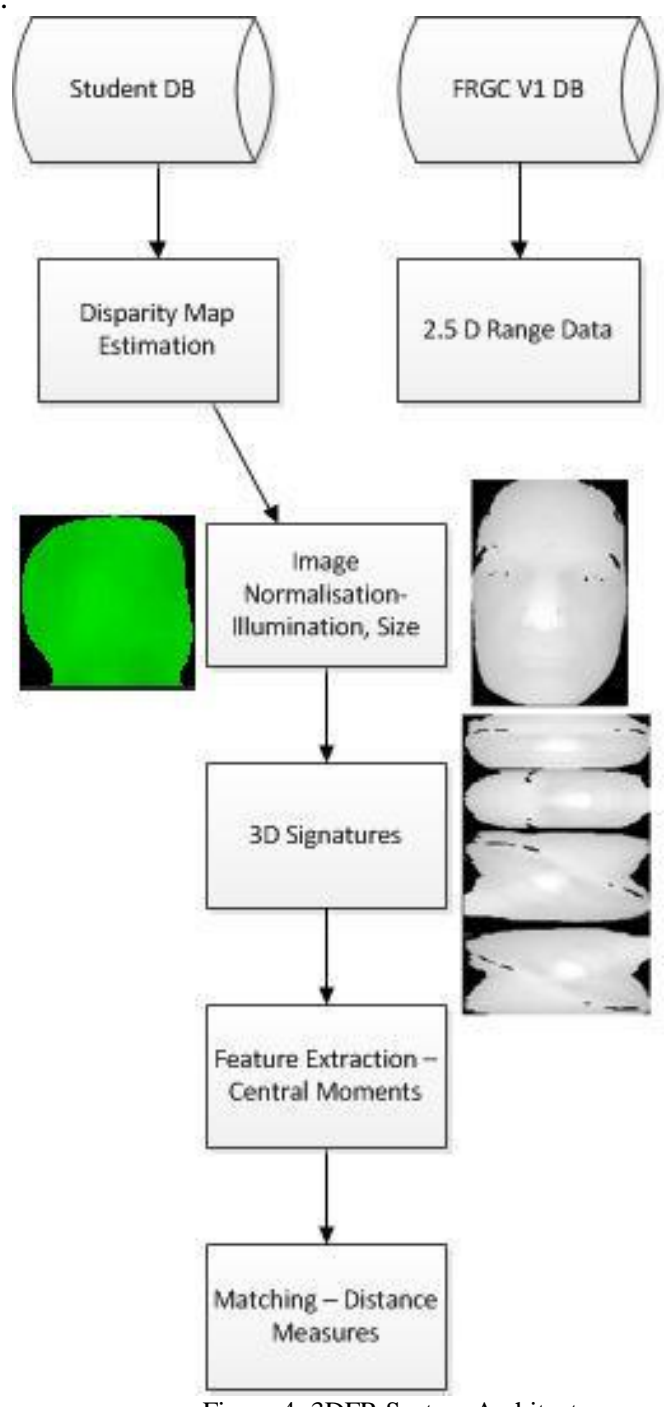

Figure 4: 3DFR System Architecture

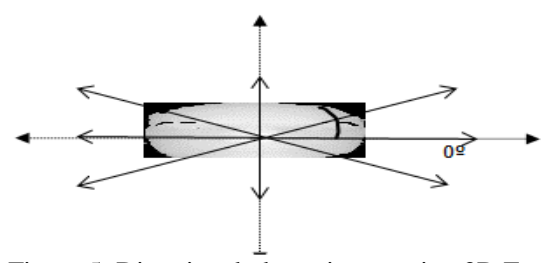

Figure 5: Directional planes intersecting 3D Face Image

TABLE II. DIMENSIONALITY OF FEATURE SETS BASED ON THE DB AND MODEL REPRESENTATION 


\section{EXPERIMENTAL SETUP AND RESULTS}

In Setions $\mathrm{I} A$ and $\mathrm{I} B$, two configurations were discussed namely databases used and model registrations built. In this Section, experimental results based on ROCs for Rank Vs Cumulative Match and FAR Vs FRR are determined. Using the notations defined in Setions $\mathrm{I} A$ and $\mathrm{I} B$, performance evaluation is carried out:

\section{A. Performance Analysis-Rank Vs Cumulative Match}

In the following tables, a set of notations for chosen angles of intersection are used:

D45 $\rightarrow$ Diagonal $45^{\circ} ; \mathrm{H} \rightarrow$ Horizontal $0^{\circ} ; \mathrm{V} \rightarrow$ Vertical $90^{\circ}$; D135 $\rightarrow$ Diagonal $135^{\circ}$; D135H $\rightarrow \Theta_{2}$ and so on. Tables III and IV relate to $\mathrm{DB}_{2}$ and in particular average Vs. individual model representations. The transient response, typically up to rank 5 , indicates low scores of match due to the high criteria of top ranking. The Cut-off is the rank beyond which a steady state response (SSR) is reached and the scores reach saturation (stable). The following configurations are considered:

\section{a) (Mind, $\left.D B_{2}\right)$ - Model-Individual, DB-FRVT (TABLE} III, Fig.6(a)).

Considering the transient performance, combinatorial features provide top $(<5)$ ranked results. Particularly poor performers are the diagonal signatures. VH combination produces a very high score. Typical Cut-off and SSR occur at ranks 10 and above and the system achieves very high scores of match at these points. Some of the higher order combinations of signatures take longer to reach SSR namely, VD135 and D45D135H. In general. Higher order combinations perform well.

\section{b) ( $\left.M_{\text {avg }}, D B_{2}\right)$ - Model-Average, DB-FRVT (TABLE IV,} Fig.6(b)).

In contrast to the individual model, the transient response of diagonal signatures of the avergae model reaches a very high score of match especially with the higher-order signatures. The cut-off points for SSR is reached at much earlier a stage as with VD45 and VD135H combinations. In terms of the overall performance with FRVT dataset, the average model performs better compared to the individual model with high scores of match.

c) (Mvg, DB $\left.{ }_{1}-7.5\right)$ - Model-Average, DB-Student with lens measurement 7.5mm (Table V, Fig.7(e-h)).

$\Theta=1$, D135 performs the worst. V is the best performer.

$\Theta=2$, VD135 performs the worst. VH is the best performer. $\Theta=3$ and 4 , overall a good performance is demonstrated.

d) (Mvg, DB I $_{1}$ 12.5)- Model-Average, DB-Student with lens measurement $12.5 \mathrm{~mm}$ (Table $\mathrm{V}$ ).

The performance is far superior to $7.5 \mathrm{~mm}$ lens and within-class feature deviation $(\boldsymbol{\sigma})$ performances are minimal in this case due to the high resolution produced by the $12.5 \mathrm{~mm}$ lens.
TABLE III: $\left(M_{\text {ind }}, D B_{2}\right)$ - ROC-Rank Vs Cumulative Match

\begin{tabular}{|l|l|l|l|l|l|}
\hline $\begin{array}{l}\text { Response } \\
\text { Match } \rightarrow\end{array}$ & \multicolumn{2}{|l|}{ Transient } & \multicolumn{2}{l|}{ Cutoff } & $\begin{array}{l}\text { Steady } \\
\text { State }\end{array}$ \\
\hline $\boldsymbol{\Theta} \downarrow$ & Score & Rank & Score & Rank & Score \\
\hline $\mathrm{H}$ & 065 & 5 & 0.89 & 10 & 0.92 \\
\hline V & 0.77 & 5 & 0.94 & 10 & 0.97 \\
\hline D45 & 0.5 & 5 & 0.825 & 10 & 0.9 \\
\hline D135 & 0.12 & 5 & 0.35 & 12 & 0.5 \\
\hline D45D135 & 0.5 & 5 & 0.75 & 16 & 0.9 \\
\hline D45H & 0.64 & 5 & 0.95 & 15 & 1 \\
\hline D135H & 0.45 & 5 & 0.75 & 15 & 0.95 \\
\hline VD45 & 0.7 & 5 & 0.94 & 11 & 0.9 \\
\hline VD135 & 0.62 & 5 & 0.9 & 20 & 0.98 \\
\hline VH & 0.88 & 5 & 0.976 & 10 & 0.977 \\
\hline VD135H & 0.74 & 5 & 0.97 & 9 & 0.98 \\
\hline VHD45 & 0.78 & 5 & 0.956 & 12 & 0.99 \\
\hline D45D135H & 0.62 & 5 & 0.87 & 16 & 0.99 \\
\hline VD45D135 & 0.75 & 5 & 0.9 & 20 & 0.98 \\
\hline VD45D135H & 0.78 & 5 & 0.98 & 11 & 0.995 \\
\hline
\end{tabular}

TABLE IV: $\left(\mathrm{M}_{\text {avg }}, \mathrm{DB}_{2}\right)$ - ROC--Rank Vs Cumulative Match

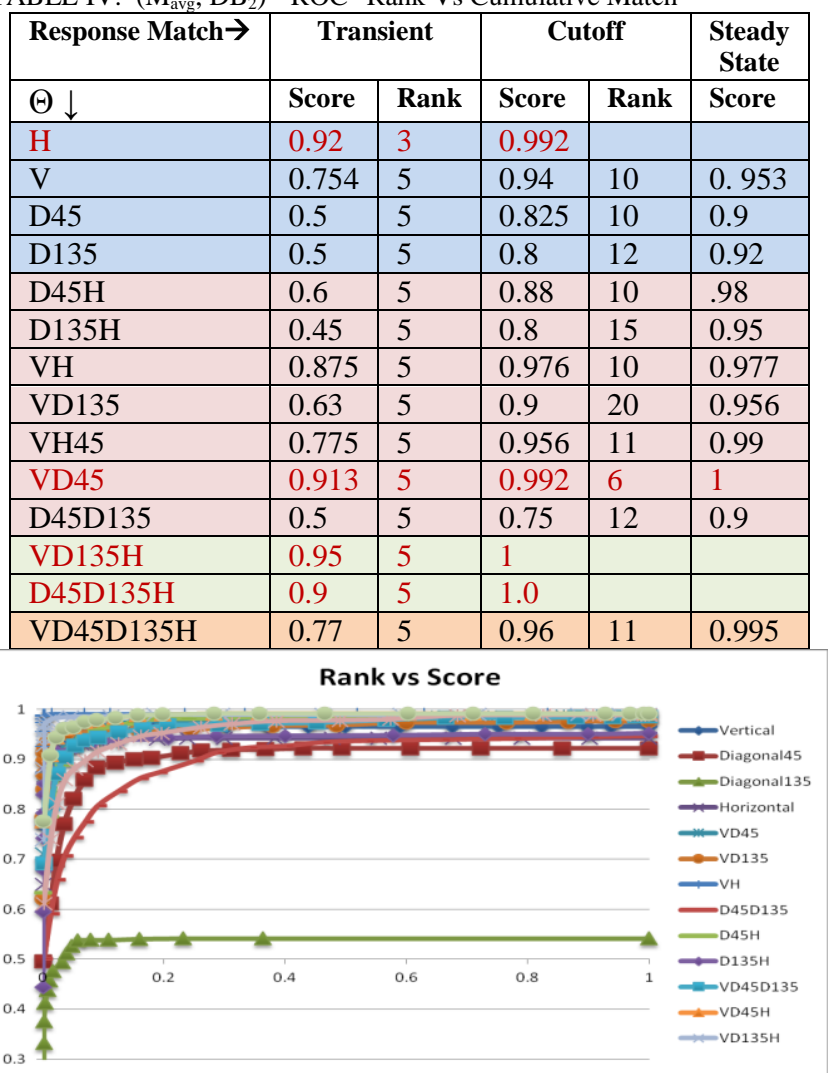

Figure 6(a): $\left(\mathrm{M}_{\mathrm{ind}}, \mathrm{DB}_{2}\right)$

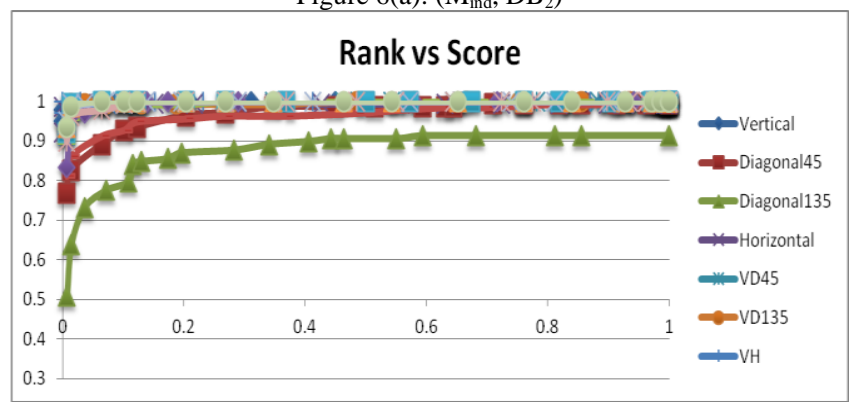

Figure 6(b): $\left(\mathrm{M}_{\mathrm{avg}}, \mathrm{DB}_{2}\right)$ 


\section{B. Performance Analysis-Equal Error Rate (EER)}

The perfomance analysis in terms of the equal error rate (EER) is carried out by separting the signatures into groups based on single or multi-angle intersections (multi-modal). Note that the $\mathrm{X}$-axis has a scaling factor of 20 in Fig7. For uni-modal signatures, i.e., $\Theta=1$, it is inferred that the diagonal signatures at $45^{\circ}, \mathrm{D} 45$ does not perform well. At rank 1, the score of match, $M_{1}(\mathbf{E E R})=0.6$, EER $\sim=0.38$. Fig.7(b-d) show the performance with signatures when $\Theta=2,3,4$ respectively. The trend plot for equal-error-rate, $M($ EER) decreases with increase in multi-modality, $\Theta$.

Similar results are echoed in the Rank Vs. Score metrics (Figs. 7(e-h)). Its trend plot $M_{1}$ (Score) decreases as well with increase in $\Theta$. For brevity, no further ROC is produced here. Results of $M_{1}$ (EER) and $M_{1}$ (Score) are shown in Tables(V-VI) and summarised below.

a) (Mind, $\left.D B_{2}\right)$ - Model-Individual, DB-FRVT (TABLE III, Fig.6(a)).

$\Theta=1$, D135 signature performs the worst and $\mathrm{V}$ the best.

$\Theta=2$, within group ROC variation $\sigma$ is small. $M_{1}$ (Score) is high.

$\Theta=3,4 \boldsymbol{\sigma}$ is very small and $M_{1}$ (Score) is very high.

Overall, the higher order signature combinations perform very well, in this case.

b) ( $\left.M_{a v g}, D B_{2}\right)$ - Model-Average, DB-FRVT (TABLE IV, Fig.6(b)).

$\Theta=1$, D135 signature performs the worst and V the best.

$\Theta=2$, within group ROC variation $\boldsymbol{\sigma}$ is spread. $M_{1}$ (Score) is high. D45D135 performs the worst and VH the best.

$\Theta=3,4 \sigma$ is spread and $M_{1}$ (Score) is average.

For $\mathrm{DB}_{2}$, the average model does not perform as well as the individual model representation.

c) (Mavg, DB $\left.{ }_{1}-7.5\right)$ - Model-Individual, DB-Student with lens measurement 7.5mm (Table VI, Fig.7(a-d))

$\Theta=1$, V performs the best while D135 performs the worst. Within group, ROC is widespread.

$\Theta=2$, VH performs best and $\mathrm{D} 135 \mathrm{H}$ performs the worst. ROC is widespread.

$\Theta=3,4$, VD45H performs the best and D45D135H performs the worst. Within group, ROC is better, less spread than low order combinations.

d) (Mavg, DB-12.5)-Model-Average, DB-Student with lens measurement $12.5 \mathrm{~mm}$ (Table VI)

The ROCs within groups are clustered together. The EER performance is far better than using $7.5 \mathrm{~mm}$ lens.

TABLE V: Performance Evaluation --Rank Vs Cumulative Match, $M_{1}$ (Score)

\begin{tabular}{|c|c|c|c|c|c|}
\hline & Parameters $\downarrow$ O $\rightarrow$ & $\mathbf{1}$ & $\mathbf{2}$ & $\mathbf{3}$ & $\mathbf{4}$ \\
\hline 1 & $\mathrm{M}_{\text {avg }}, \mathrm{DB}_{1}-7.5$ & 0.3 & 0.56 & 0.62 & 0.66 \\
\hline 2 & $\mathrm{M}_{\text {avg }}, \mathrm{DB}_{1}-12.5$ & 0.71 & 0.82 & 0.85 & 0.87 \\
\hline 3 & $\mathrm{M}_{\text {avg }}, \mathrm{DB}_{2}$ & $(0.5,0.92)$ & $(0.8,1)$ & $(0.9,1)$ & $(0.92,1)$ \\
\hline 4 & $\mathrm{M}_{\text {ind }}, \mathrm{DB}_{2}$ & $(0.3 \mathrm{D}, 0.9)$ & $(0.42,1)$ & $(0.6,0.9)$ & 0.78 \\
\hline
\end{tabular}

TABLE VI: Performance Evaluation - $M_{1}$ (EER)

\begin{tabular}{|c|c|c|c|c|c|c|}
\hline & Parameters $\downarrow$ O $\rightarrow$ & $\mathbf{1}$ & $\mathbf{2}$ & $\mathbf{3}$ & $\mathbf{4}$ & $\boldsymbol{\sigma}$ \\
\hline 1 & $\mathrm{M}_{\text {avg }}, \mathrm{DB}_{1}-7.5$ & 0.38 & 0.345 & 0.34 & 0.365 & spread \\
\hline 2 & $\mathrm{M}_{\text {avg }}, \mathrm{DB}_{1}-12.5$ & 0.23 & 0.18 & 0.18 & 0.19 & v. small \\
\hline 3 & $\mathrm{M}_{\text {avg }}, \mathrm{DB}_{2}$ & 0.8 & 0.05 & 0.08 & 0.08 & spread \\
\hline 4 & $\mathrm{M}_{\text {ind }}, \mathrm{DB}_{2}$ & 0.25 & 0.18 & 0.28 & 0.28 & v.spread \\
\hline
\end{tabular}

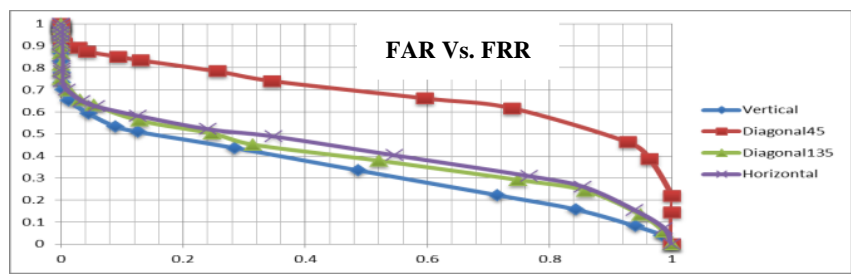

Figure 7(a): $\left(\mathrm{M}_{\mathrm{avg}}, \mathrm{DB}_{1}-7.51\right): \Theta=1, \mathrm{M}_{1}=0.6, \mathrm{EER}=0.38$

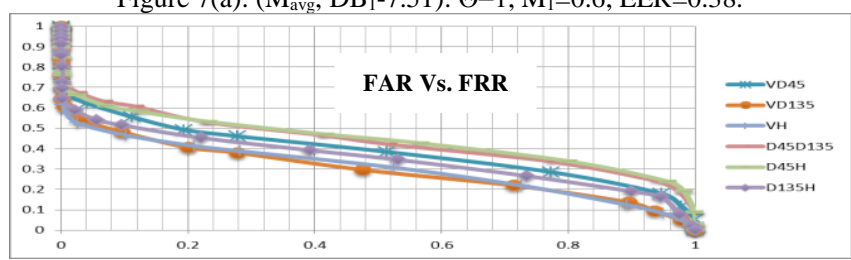

Figure 7(b): $\left(\mathrm{M}_{\text {avg }}, \mathrm{DB}_{1}-7.51: \Theta=2,, \mathrm{M}_{1}=0.5, \mathrm{EER}=0.345\right.$

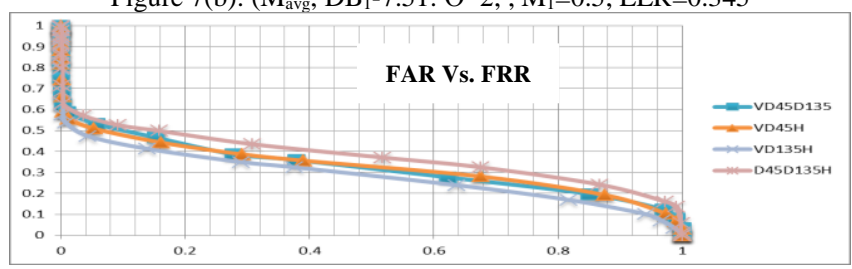

Figure 7(c): $\left(\mathrm{M}_{\mathrm{avg}}, \mathrm{DB}_{1}-7.51: \Theta=3, \mathrm{M}_{1}=0.46, \mathrm{EER}=0.34\right.$

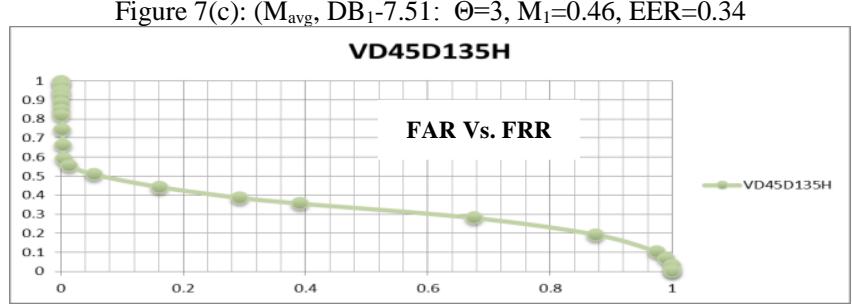

Figure 7(d): $\left(\mathrm{M}_{\mathrm{avg}}, \mathrm{DB}_{1}-7.51\right): \Theta=3, \mathrm{M}_{1}=0.5, \mathrm{EER}=0.365$

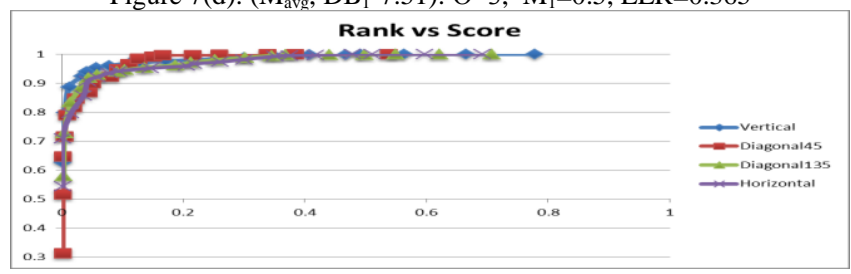

Figure 7(e): $\left(\mathrm{M}_{\text {avg }}, \mathrm{DB}_{1}-7.51\right): \Theta=1, \mathrm{M}_{1}=0.6, \mathrm{EER}=0.38$

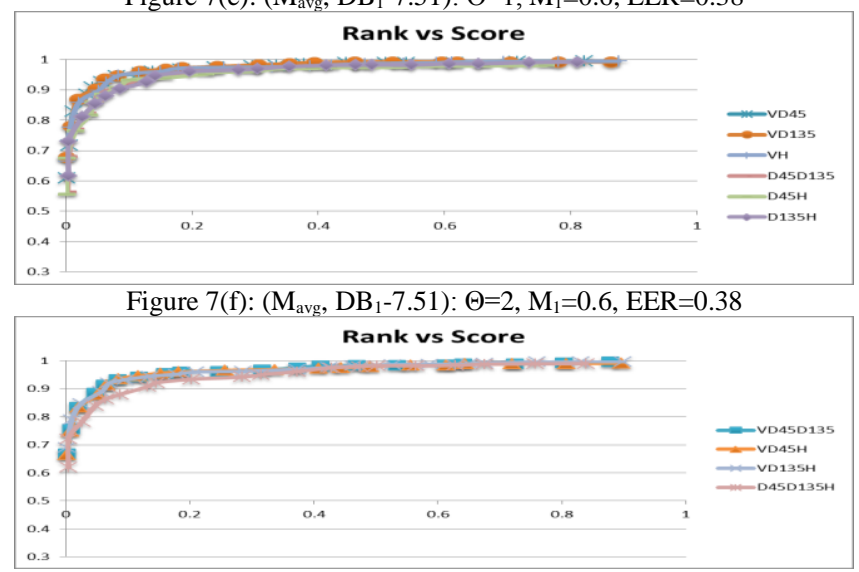

Figure 7(g): $\left(\mathrm{M}_{\text {avg }}, \mathrm{DB}_{1}-7.51\right): \Theta=3, \mathrm{M}_{1}=0.6, \mathrm{EER}=0.38$ 


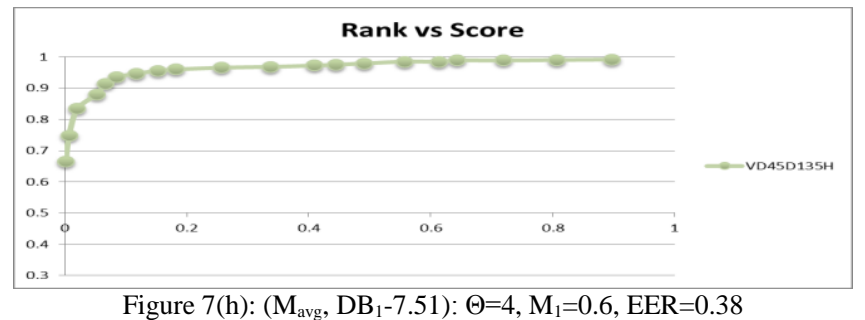

Figure 7(h): $\left(\mathrm{M}_{\mathrm{avg}}, \mathrm{DB}_{1}-7.51\right): \Theta=4, \mathrm{M}_{1}=0.6, \mathrm{EER}=0.38$

\section{SUMMARY}

In this work, two databases and two model configurations were considered for performance evaluation. Higher -order signature combinations were used as primary features and their usefulness tested. The following are noted:

- The average model performs better than the individual model in respect of $M_{1}$ (Score) but not so good in reducing the EER.

- Higher order signature combinations are useful in reducing the EER and increasing the matching score.

- Not all features are useful especially D135 signature and acts as an outlier to the ROC.

- Uni-modal signatures perform very well especially the Vertical signatures across the board of testing.

Further work is being carried out in the following directions:

- Providing generalization results whereby the data is partitioned into learning and testing and are mutually exclusive. This performance evaluation will determine the ability to work with unseen data.

- Providing individual model analysis for the student DB.

- Partitioning the database into sub-databases and performing matching to reduce EER and increase $M_{1}$ (Score). This is particularly a useful criterion in handling large databases.

- $\quad$ Two-stage matching where LDA is first applied to get a subset of matches followed by FLDA to work on the subset. This, mechanism has been tested on a smaller database in [16] showing very promising results, which is to be extended to the databases considered here.

\section{REFERENCES}

[1] P. Sharma and M. Goyani, "3d face recognition techniques - a review," International Journal of EnginEERing Research and Applications (IJERA), vol. 2, no. 787-798, Feb 2012

[2] S. Huq, B. Abidi, S. G. Kong, and M. Abidi, "A survey on 3d modeling of human faces for face recognition," in 3D Imaging for Safety and Security, ser. Computational Imaging and Vision, A. Koschan, M. Pollefeys, and M. Abidi, Eds. Springer Netherlands, 2007, vol. 35, pp. 25-67.

[3] Y. Wang, J. Liu, and X. Tang, "Robust 3d face recognition by local shape difference boosting," Pattern Analysis and Machine Intelligence, IEEE Transactions on, vol. 32, no. 10, pp. $1858-1870$, oct. 2010.

[4] D. Huang, K. Ouji, M. Ardabilian, Y. Wang, and L. Chen, "3d face recognition based on local shape patterns and sparse representation classifier," in Advances in Multimedia Modeling, ser. Lecture Notes in Computer Science, K.-T. Lee, W.-H. Tsai, H.-Y. Liao, T. Chen, J.W. Hsieh, and C.-C. Tseng, Eds. Springer Berlin / Heidelberg, 2011, vol. 6523, pp. 206-216.

[5] H. Tang, Y. Sun, B. Yin, and Y. Ge, "3d face recognition based on sparse representation,” J. Supercomput., vol. 58, pp. 84-95, October 2011.

[6] K. Tae-Kyun and J. Kittler, "Design and fusion of pose invariant face-identification experts," Circuits and Systems for Video Technology, IEEE Transactions on, vol. 16, no. 9, pp. $1096-1106$, Sept. 2006.

[7] F. Hajati, A. Raie, and Y. Gao, "Pose-invariant multimodal (2d+3d) face recognition using geodesic distance map," Journal of American Science, vol. 7, 2011.

[8] P. Xiong, L. Huang, and C. Liu, "Real-time 3d face recognition with the integration of depth and intensity images," in Proceedings of the 8th international conference on Image analysis and recognition Volume Part II, ser. ICIAR'11. Berlin, Heidelberg: Springer-Verlag, 2011, pp. 222-232.

[9] V. Nirgude, A. Gulve, and S.Waghmare, "Face recognition system using principal component analysis \& linear discriminant analysis method simultaneously with $3 \mathrm{~d}$ morphable model," in Proc. of Int. Conf. on Advances in Computing, Communication, and Networks, 2011.

[10] Bronstein, M.B., R. Kimmel, and A. Spira. 3D face recognition without facial surface reconstruction. in European Conference on Computer Vision. 2004.

[11] Gordon, G. Face Recognition Based on Depth and Curvature Features. in IEEE Computer Society Conference on Computer Vision and Pattern Recognition. 1992.

[12] Lee, J.C.a.M., E. . Matching range images of human faces. in IEEE International Conference on Computer Vision. 1990. Osaka, Japan.

[13] Beumier, C.a.A., M. Automatic Face Verification from 3D And Grey Level Clues. in 11th Portuguese Conference on Pattern Recognition. 2000

[14] Anshuman Razdan, G.F., Myung Soo-Bae and Mahesh Chaudhari State of 3D Face Biometrics for Homeland Security Applications, in National Security (Handbooks on Information Systems), T.S.R. Hsinchun Chen, Ram Ramesh, Ajay Vinze and Daniel Zeng, Editor. 2007, Elsevier. p. 73-99

[15] P. Jonathon Phillips, W. Todd Scruggs, Alice J. O'Toole, Patrick J. Flynn, Kevin W.Bowyer4, Cathy L. Schott, Matthew Sharpe, FRVT 2006 and ICE 2006 Large-Scale Results, March 200, Report NISTIR 7408, National Institute of Standards and Technology, US.

[16] R.Soodamani and Ronda Venkateswarlu, Stereo Face Recognition using Discriminant Eigenvectors, Proceedings, WSES International Conference on Speech, Signal and Image Processing 2001 (SSIP 2001), Sep.1-6, 2001, Malta, pp.2621-2626, 2001.

[17] Sokanya M.S. Al-Qatawneh, 3D Facial Feature Extraction and Recognition, Ph.D. Thesis, School of Computing, Informatics and Media, University of Bradford, UK, 2010.

[18] Dan Hua, Image Illumination Normalisation for 2D/3D Face Images, M.Sc. thesis dissertation, Supervisor: Soodamani Ramalingam, School of EnginEERing and Technology, University of Hertfordshire, Apr. 2012. 